\title{
Identification of female carriers for Duchenne and Becker muscular dystrophies using a FISH-based approach
}

\author{
Azra H Ligon ${ }^{1,2}$, Catherine D Kashork ${ }^{1}$, C Sue Richards ${ }^{1}$ and Lisa G Shaffer ${ }^{1}$ \\ ${ }^{1}$ Department of Molecular and Human Genetics, Baylor College of Medicine, Houston, TX
}

\begin{abstract}
Duchenne muscular dystrophy (DMD) and Becker muscular dystrophy (BMD) are X-linked recessive neuromuscular diseases caused by dystrophin gene mutations. Deletions, or more rarely duplications, of single or multiple exons within the dystrophin gene can be detected by current molecular methods in approximately $65 \%$ of DMD patients. Mothers of affected males have a two-thirds chance of carrying a dystrophin mutation, whilst approximately one-third of affected males have de novo mutations. Currently, Southern blot analysis and multiplex PCR directed against exons in deletion hot spots are used to determine female carrier status. However, both of these assays depend on dosage assessment to accurately identify carriers since, in females, the normal $X$ chromosome is also present. To obviate quantitation of gene dosage, we have developed exon-specific probes from the dystrophin gene and applied them to a screen for potential carrier females using fluorescence in situ hybridization (FISH). Cosmid clones, representing 16 exons, were identified and used in FISH analysis of DMD/BMD families. Our preliminary work has identified multiple, informative probes for several families with dystrophin deletions and has shown that a FISH-based assay can be an effective and direct method for establishing the DMD/BMD carrier status of females. European Journal of Human Genetics (2000) 8, 293-298.
\end{abstract}

Keywords: Duchenne muscular dystrophy; Becker muscular dystrophy; carrier detection; FISH; laboratory validation; diagnostic testing

\section{Introduction}

Duchenne muscular dystrophy (DMD) is an X-linked recessive disorder that affects $1 / 3500$ liveborn males. ${ }^{1}$ Most affected males appear healthy at birth but may achieve certain developmental milestones (such as walking) at time points later than unaffected children. By five years of age, proximal muscle weakness is apparent and decreased muscle strength causes eventual loss of ambulation by early adolescence. The disease progresses to include distal muscle loss

Correspondence: Lisa G Shaffer, PhD, Department of Molecular and Human Genetics, Baylor College of Medicine, One Baylor Plaza, Room 15E, Houston, Texas 77030, USA. Tel: +1713 798 5919; Fax: +1713 798 3157; E-mail: Ishaffer@bcm.tmc.edu

${ }^{2}$ Current address: Department of Pathology, Brigham and Women's Hospital/ Harvard Medical School, Boston, MA, USA

Received 12 July 1999; revised 22 November 1999; accepted

24 November 1999 and respiratory and/or cardiac failure and ultimately causes death. ${ }^{1}$

Mutations in the dystrophin gene, which maps to Xp21, are responsible for DMD. ${ }^{2}$ Dystrophin is an unusually large gene, spanning at least 79 exons. Approximately $60 \%$ of mutations in the dystrophin gene, leading to either Duchenne muscular dystrophy or the milder, allelic Becker muscular dystrophy (BMD), are deletions. ${ }^{3}$ Frameshift deletions result in DMD (with no functional dystrophin protein produced), while deletions that maintain the reading frame produce the BMD phenotype (partially functional dystrophin present). Within the dystrophin gene, these deletions tend to cluster in hot spots, ${ }^{3}$ two of which map towards the 5 ' end of the gene (encompassing exons 3-7) and the central portion of the gene (encompassing exons43-51). Approximately two-thirds of mothers of affected males with known deletions are themselves asymptomatic carriers of DMD 
(about $30 \%$ of males with DMD represent de novo mutations).

The current methods most commonly used for molecular diagnosis of deletions at the DMD/BMD locus involve exonspecific PCR and Southern analysis to distinguish between deleted and nondeleted individuals. These molecular diagnostic techniques are excellent for detecting dystrophin deletions in males. However, for females, who possess two $X$ chromosomes, the detection of deletions (establishing carrier status) can be problematic, since current methods rely on assessing dosage between regions of the gene. In principle, a carrier female should show half the normal signal intensity by Southern analysis, however this determination can be somewhat subjective and the ability to establish a dosage difference might vary from laboratory to laboratory. While quantitative PCR has been used to address this problem, ${ }^{4}$ the issue of subjective interpretation remains.

In contrast, FISH analysis for chromosomal deletions is a well-established technique that has been, and continues to be, used to identify a number of deletion symdromes. ${ }^{5}$ In limited studies, probes ranging in size from cDNAs to genomic inserts cloned into yeast artificial chromosomes (YACS) have been successfully applied towards detection of DMD deletions. ${ }^{6-9}$ FISH offers a number of advantages, including:

(1) dosage assessment is alleviated,

(2) radioisotopes are unnecessary because signal detection is achieved through fluorescence, and

(3) direct visual assessment of the presence or absence of a deletion (ie either one or two fluorescent signals present on the homologous chromosome).

Thus, FISH is a qualitative approach, instead of a quantitative-based data interpretation.

In the first reported study, FISH analysis was performed to assess DMD patients whose deletions included exon 45 of dystrophin and their female relatives. ${ }^{6} \mathrm{~A}$ set of three cosmid clones spanning the region including exon 45 was used to confirm the carrier status in all individuals tested. This study showed the utility of a fluorescence-based approach for which scoring of the results is relatively rapid and direct. In a more recent study, the feasibility of offering a panel of exonspecific cosmid probes was assessed. ${ }^{10}$ Six probes were used on cell lines established from eight males and three females, representing eight, known unique deletions. FISH analyses demonstrated deletions in all cases, confirming previous molecular analyses. In a different approach, Rosenberg et $\mathrm{al}^{11}$ assessed the most frequently deleted exons of the dystrophin gene and devised a panel of cosmid probes for FISH. Using systematic screening, deletions were identified appropriately in 21 heterozygous females and nine control females. We report the use of a new set of FISH probes to screen 24 families with a history of DMD or BMD.

\section{Materials and methods Isolation of exon-specific cosmids}

To obtain probes for screening purposes, primer sets from a dystrophin multiplex PCR assay were used to amplify exons of interest from human genomic DNA as described. ${ }^{12,13}$ Products were obtained for the following exons: 3-6; 8, 12, $13,17,19,43,44,45,48,50,51$, and 60. The amplified products were gel-purified and the DNA was radiolabeled using the method of random priming (Stratagene Prime-It II Kit, La Jolla, CA, USA.) ${ }^{14}$ An X chromosome arrayed cosmid library (LLOXNCO1'U') was plated and transferred to a total of 15 nylon membranes and screened sequentially with the appropriate exon-specific PCR-generated probes. Membranes were prehybridized $6 \mathrm{~h}$ to overnight in $6 \mathrm{X} \mathrm{SSC} / 5 \mathrm{X}$ Denhardt's $/ 0.5 \% \mathrm{SDS} / 0.5 \mu \mathrm{g} / \mu \mathrm{l}$ salmon sperm DNA at $65^{\circ} \mathrm{C}$. Radiolabeled probes were added at a final concentration of $1-2 \times 10^{6} \mathrm{cpm} / \mathrm{ml}$ and hybridized overnight. Membranes were washed twice for $15 \mathrm{~min}$ each in $2 \mathrm{X} \mathrm{SSC} / 1 \% \mathrm{SDS}$ at $65^{\circ} \mathrm{C}$ and once for $20 \mathrm{~min}$ in $1 \mathrm{X} \mathrm{SSC} / 0.1 \% \mathrm{SDS}$ at $65^{\circ} \mathrm{C}$. Moist membranes were sealed in plastic wrap, placed next to Kodak XAR film using intensifying screens, and exposed at $-80^{\circ} \mathrm{C}$ for $8 \mathrm{~h}$ to 5 days. Positive clones were identified, streaked on LB-kanamycin plates, cultured and DNA extracted following standard protocols. ${ }^{15}$

\section{Characterization of cosmids}

Identified cosmids were characterized to ensure the presence of the target exon as well as the absence of any neighboring exons. With the same primers used previously for PCR, DNA from each cosmid clone was amplified to test for the presence of the desired exon. Cosmids identified to contain the same exon were digested with a series of restriction enzymes to identify differential patterns which may indicate either unique or identical/overlapping clones. Selected clones were then screened by PCR for neighboring exons. When possible, clones containing only a single exon were used for FISH. A single, representative cosmid was chosen for FISH when multiple cosmids were determined to include the same exon.

\section{FISH analysis of DMD families}

Blood samples were collected from 24DMD/BMD families who were interested in participating in the research and development of a new diagnostic tool. Samples were cultured, metaphase chromosomes were obtained, and slides were prepared according to standard cytogenetic protocols. To confirm hybridization of the target probe in males, metaphases from a normal female control pellet were placed to the right-hand side of the affected male sample on the microscope slide. Cosmid probes were labeled with digoxigenin by the method of nick-translation as previously described. ${ }^{16}$ An alpha-satellite $X$ chromosome probe was purchased labeled with biotin (Oncor; Gaithersburg, MD or Vysis, Inc., Downers Grove, IL, USA) and $0.5 \mu$ l was added to each slide to aid in the identification of the $X$ chromosomes. 
Final probe concentration of each cosmid was $35-50 \mathrm{ng} / \mu \mathrm{l}$. The FISH was performed and signals were detected as previously described for two-color FISH. ${ }^{16}$

\section{Visualization and scoring of cases}

All metaphase chromosomes were visualized and scored using a Zeiss Axiophot fluorescence microscope equipped with a triple band-pass filter, allowing for the simultaneous visualization of red and green signals. Ten to 20 metaphases per individual were analysed with images captured using a Perceptive Scientific Instruments Powergene 810 probe system (League City, TX). Enhanced images were printed on a Tektronix Color/Monochrome Phasar II SDX printer. For affected males, a deletion was evident when there was absence of the cosmid signal and presence of only the centromeric signal (Figure1A). Once a probe(s) was identified as deleted in an affected male, the same probe(s) would be used to screen female relatives in the family. Female carriers show only one signal for the dystrophin cosmid on the normal $\mathrm{X}$ chromosome and two centromeric signals (one on each homologue) (Figure 1B). In contrast, a non-carrier female would show two cosmid signals and two centromeric signals (Figure1C). In some families, the affected male was not available for analysis. Whenever possible, the FISH probe was demonstrated as deleted in a known carrier, before testing other female relatives.

\section{Patient population}

Families with a history of DMD were ascertained through genetic centers and muscular dystrophy clinics in the United
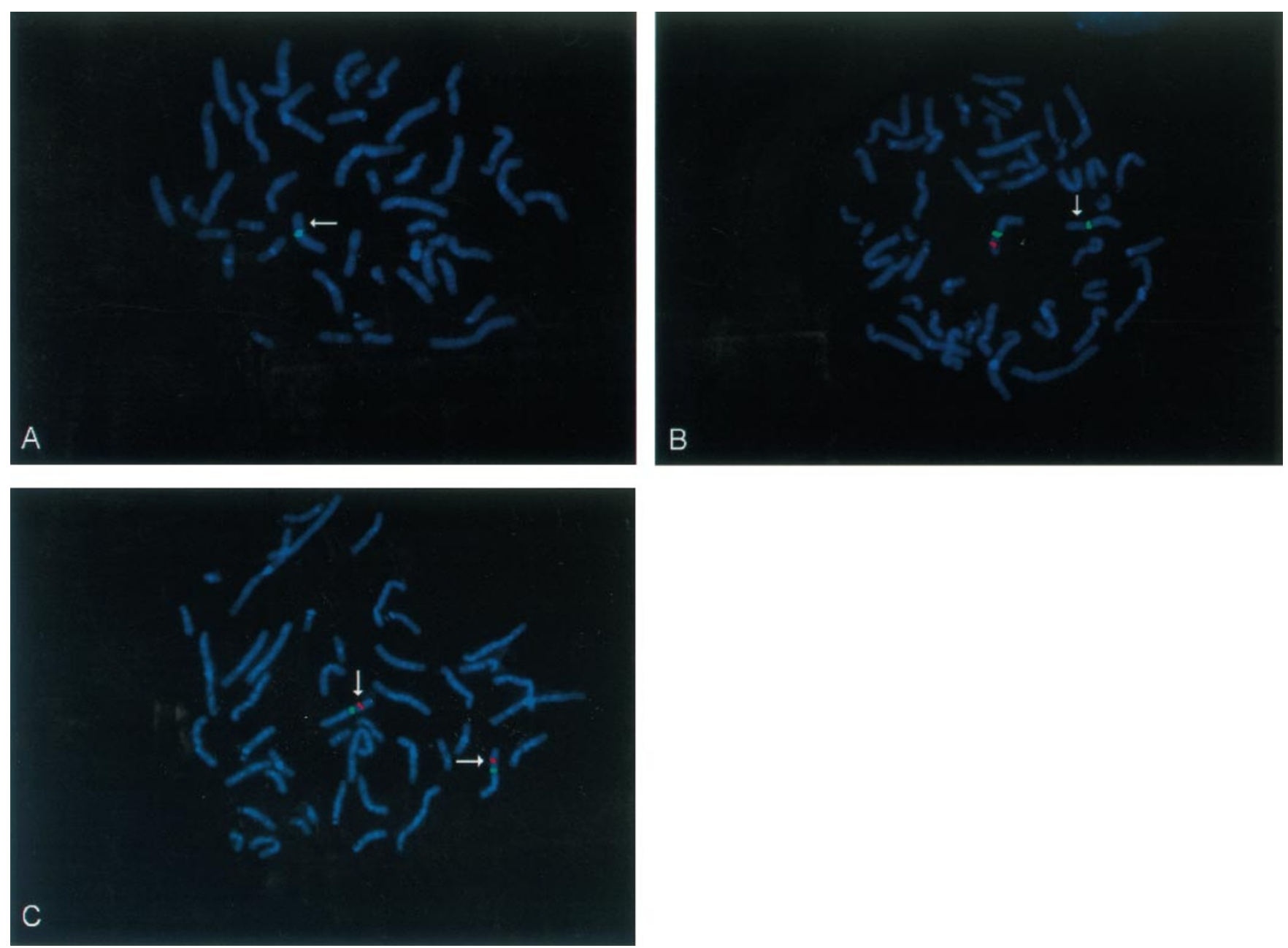

Figure 1 Representative FISH results for three families. A FISH using cosmid 36E6, containing exons 3-6, on the affected DMD male from Family 1 showed only the green centromeric signal (arrow) and absence of the cosmid signal, demonstrating a deletion on his X chromosome. B FISH using cosmid 138E6, containing exon 12, on the daughter of a BMD male in Family 13 showed a deletion of the cosmid signal on one X chromosome (arrow), demonstrating that she is a carrier. C FISH using cosmid 141G11, containing exon 44, on the sister in Family 6 showed a normal hybridization pattern to both $X$ chromosomes (arrows), indicating that she is not a carrier of the DMD deletion. 
States. Thirty-six individuals, from 24 families, were studied using FISH. Of these, eight families had affected males with known molecular deletions available for study. Of the remaining 16 families, for whom affected males were not available, prior molecular results were available for 17 of 19 females studied using FISH.

\section{Results}

The screening of the $X$ chromosome specific cosmid library identified 13 dystrophin exon-specific probes (Table1). For the eight mal es tested using FISH, the expected deletion was demonstrated in every case (Table2). None of the nine corresponding female relatives demonstrated a deletion by FISH. Thus, there was $100 \%$ concordance with the available molecular diagnostic results (5/9females tested by standard molecular methods). Of the 19 females studied using FISH for whom no affected male was available, prior molecular results were available for 17 cases. For these, deletions were found using FISH in seven, demonstrating complete agreement with the prior molecular results. For two mothers, molecular analysis for carrier detection had not been performed and no deletions were detected using FISH. Therefore, for these women, it is uncertain if the probes used fall within the deleted region and these women are truly not carriers or,

Table 1 Exon-specific cosmid probes identified and used in this study

\begin{tabular}{lc}
\hline Cosmid & Exons(s) \\
\hline $36 \mathrm{E} 6$ & $3-6$ \\
337 & 8 \\
$138 \mathrm{E} 6$ & 12 \\
$61 \mathrm{~F} 4$ & 13 \\
$105 \mathrm{G} 11$ & 17 \\
$101 \mathrm{H} 2$ & 19 \\
$59 \mathrm{E} 11$ & 43 \\
$141 \mathrm{G} 11$ & 44 \\
$48 \mathrm{~F} 5$ & 45 \\
222F5 & 48 \\
$40 \mathrm{H} 11$ & 50 \\
107G12 & 51 \\
22C6 & 60 \\
\hline
\end{tabular}

Table 2 Results for 24 families comparing standard diagnostic testing with FISH

\begin{tabular}{|c|c|c|c|c|c|c|}
\hline Family number & $\begin{array}{l}\text { Relationship } \\
\text { to affected }\end{array}$ & Gender & $\begin{array}{l}\text { Known } \\
\text { molecular deletions }\end{array}$ & Using FISH & FISH results & Interpretation \\
\hline \multirow[t]{2}{*}{1} & proband & male & $2-30$ & $3-6,13$ & deleted & affected \\
\hline & mother & female & none & $3-6,13$ & normal & noncarrier \\
\hline \multirow[t]{2}{*}{2} & proband & male & $49-50$ & 50 & deleted & affected \\
\hline & mother & female & none & 50 & normal & noncarrier \\
\hline \multirow[t]{2}{*}{3} & proband & male & $45-50$ & $45,48,50$ & deleted & affected \\
\hline & mother & female & none & $45,48,50$ & normal & noncarrier \\
\hline \multirow[t]{2}{*}{4} & proband & male & $46-51$ & 50 & deleted & affected \\
\hline & mother & female & none & 50 & normal & noncarrier \\
\hline \multirow[t]{3}{*}{5} & proband & male & $7-13$ & 8 & deleted & affected \\
\hline & sister & female & not tested & 8 & normal & noncarrier \\
\hline & sister & female & not tested & 8 & normal & noncarrier \\
\hline \multirow[t]{2}{*}{6} & proband & male & 44 & 44 & deleted & affected \\
\hline & sister & female & none & 44 & normal & noncarrier \\
\hline \multirow[t]{2}{*}{7} & proband & male & $45-50$ & 48 & deleted & affected \\
\hline & mother & female & not tested & 48 & normal & noncarrier \\
\hline \multirow[t]{2}{*}{8} & proband & male & $50-52$ & 51 & deleted & affected \\
\hline & mother & female & not tested & 51 & normal & noncarrier \\
\hline \multirow[t]{2}{*}{9} & mother & female & $46-48$ & 48 & deleted & carrier \\
\hline & sister & female & none & 48 & normal & noncarrier \\
\hline \multirow[t]{2}{*}{10} & mother & female & $49-50$ & 50 & deleted & carrier \\
\hline & sister & female & none & 50 & normal & noncarrier \\
\hline 11 & sister & female & none (51) & 51 & normal & noncarrier \\
\hline 12 & mother & female & $5-16$ & 13 & deleted & carrier \\
\hline 13 & daughter & female & $10-30$ & 12,19 & deleted & carrier \\
\hline 14 & mother & female & none (49-50) & 50 & normal & noncarrier \\
\hline 15 & mother & female & none (8-25) & $12,13,19$ & normal & noncarrier \\
\hline 16 & mother & female & none (49-52) & 50 & normal & noncarrier \\
\hline 17 & mother & female & none (45) & 45 & normal & noncarrier \\
\hline 18 & sister & female & none (45) & 45 & normal & noncarrier \\
\hline \multirow[t]{2}{*}{19} & grandmother & female & none (38-43) & 43 & normal & noncarrier \\
\hline & aunt & female & none (38-43) & 43 & normal & noncarrier \\
\hline 20 & mother & female & not tested (45-47) & 45 & normal & noncarrier \\
\hline 21 & mother & female & $44-50$ & $44,45,48$ & deleted & carrier \\
\hline 22 & mother & female & $48-50$ & 48,50 & deleted & carrier \\
\hline 23 & mother & female & not tested (46-48) & 48 & normal & noncarrier \\
\hline 24 & mother & female & 45 & 45 & deleted & carrier \\
\hline
\end{tabular}

anumbers in parentheses indicate the exons that are known to be deleted in an affected male in the family 
alternatively the probe may be larger than the deleted region resulting in hybridization to both $\mathrm{X}$ chromosomes, giving fal se negative results.

\section{Discussion}

Since the dystrophin gene is so large, it presents unique advantages and disadvantages in a diagnostic setting. To develop a FISH-based approach, the 79 exons in this gene suggest that many exon-specific cosmids must be obtained so that the majority of families with deletions for any exon can be identified. However, dystrophin deletions are known to cluster in hot spots. ${ }^{3,11}$ Rosenberg et $\mathrm{al}^{11}$ developed a set of FISH probes based on these hot spot regions. We have also identified cosmids for each of these hot spot regions, enabling carrier status to be elucidated for a large majority of mothers and female relatives of boys with deletions in the dystrophin locus. Additionally, we developed probes outside the deletion hot spots that have allowed for deletion identification in a larger number of families (families5, 13, 15, and 19). Family 19, and possibly families 9 and 23, would not have been identified using the probe set developed by Rosenberg et al. ${ }^{11}$

Probes were validated through comparison with molecular results obtained using established and accepted methods of diagnostic testing. In every case, our molecular cytogenetic data were in complete agreement with the known molecular data ( $n=30$ individuals). By analyzing potential carrier females in a blinded fashion (ie with no knowledge of molecular testing results prior to scoring of the FISH results), the sensitivity of the assay (ie the probability that a true deletion is scored as such) could be determined and was found to be $100 \%$ in this investigation. In addition, each known non-deleted case was confirmed by the FISH assay, thus providing a specificity of $100 \%$ in our samples. Both these parameters provide a measure of the robustness of this FISH-based approach.

Our results confirm those obtained from a recent pilot study performed by our laboratory ${ }^{10}$ and those reported previously describing the use of FISH analysis for DMD. ${ }^{6-9,11}$ In each case, the carrier status (or disease status) could be correctly identified using FISH. However, there are some restrictions to the use of a FISH-based approach to carrier testing. First, most DMD patients that have dystrophin gene deletions (about $60 \%$ ) or intragenic duplications (about $5 \%$ ) can be tested using FISH; those with point mutations will have to rely on molecular analysis or linkage assessment to establish carrier status. Second, it is expected that certain deletions will be smaller in size than the probe used for testing. In these instances, a particular exon may be deleted but surrounding intronic sequences may not, so a deleted individual may show a normal hybridization pattern and be scored as not deleted (normal). Third, not all patients with deletion of the same exons will have the identical deletion endpoints. Since this variability will undoubtedly exist between families, a probe that is informative within a given family may not be informative for others. It is precisely for this reason that all probes to be used in a family must first be shown to be deleted in an individual who has had a deletion identified through prior molecular testing (affected males or carrier females).

It would be inappropriate to suggest that FISH be used in place of either multiplex PCR or Southern analysis to confirm or make a diagnosis of DMD/BMD in an affected male, or in a family in which no individual has had a prior molecular analysis. It is not possible to date, nor feasible with current FISH technologies, to analyze all dystrophin exons using FISH. Instead, we have demonstrated that FISH is an attractive, alternative approach to screen female rel atives of a known affected male with a molecular diagnosis of DMD or BMD. The ultimate goal of this work is to isolate probes representing all, or at least the majority, of the exons in the dystrophin gene so that FISH analysis can be performed for the majority of DMD families who carry deletions. These probes may be amenable to new FISH technologies, such as simultaneous detection devices ${ }^{17}$ and micro-array technologies $^{18}$ to allow for rapid and accurate assessment for deletions in males and females.

\section{Acknowledgements}

We thank the families who participated in this study, the clinicians who collected and submitted specimens, and Jessica Wu, Aimee Spikes, and Kay Atkins for their expert FISH analyses (Baylor College of Medicine, Houston, TX). This study was supported in part by a grant from the Muscular Dystrophy Association (to LGS).

\section{References}

1 Emery AE: Duchenne muscular dystrophy. In: Oxford Monographs on Medical Genetics, vol.15. Oxford University Press: Oxford, 1987.

2 Koenig M, Hoffman EP, Bertelson CJ, Monaco AP, Feener C, Kunkel LM: Complete cloning of the Duchenne muscular dystrophy (DMD) CDNA and preliminary genomic organization of the DMD gene in normal and affected individuals. Cell 1987; 50: 509-517.

3 Koenig $M$, Beggs $A H$, Moyer $M$ et al: The molecular basis for Duchenne versus Becker muscular dystrophy: correlation of severity with type of deletion. Am J Hum Genet 1989; 45: 498-506.

4 Mansfield ES, Robertson JM, Lebo RV et al: Duchenne/Becker muscular dystrophy carrier detection using quantitative PCR and fluorescence-based strategies. Am J Med Genet 1993; 48: 200-208.

5 Shaffer LG: Diagnosis of microdeletions syndromes by fluorescence in situ hybridization (FISH). In: Dracopoli NC, Haines JL, Korf BR et al (eds). Current Protocols in Human Genetics. John Wiley: New York, 1995 Supplement 6, Unit 8.10, pp 1-13.

6 Ried T, Mahler V, Vogt $P$ et al: Direct carrier detection by in situ suppression hybridization with cosmid clones of the Duchenne/ Becker muscular dystrophy locus. Hum Genet 1990; 85: 581-586.

7 Voit T, Neuen-Jacob E, Mahler V, Jauch A, Cremer M: Somatic mosaicism for a deletion of the dystrophin gene in a carrier of Becker muscular dystrophy. Eur J Pediatr 1992; 151: 112-116. 
8 Tocharoentanaphol C, Cremer M, Schrock E et al: Multicolor fluorescence in situ hybridization on metaphase chromosomes and interphase Halo-preparations using cosmid and YAC clones for the simultaneous high resolution mapping of deletions in the dystrophin gene. Hum Genet 1994; 93: 229-235.

9 Bunyan DJ, Crolla JA, Collins AL, Robinson DO: Fluorescence in situ hybridisation studies provide evidence for somatic mosaicism in de novo dystrophin gene deletions. Hum Genet 1995; 95: 43-45.

10 Voskova-Goldman A, Peier A, Caskey CT, Richards CS, Shaffer LG: DMD-specific FISH probes are diagnostically useful in the detection of female carriers of DMD gene deletions. Neurology 1997; 48: 1633-1638.

11 Rosenberg C, Navajas L, Vagenas DF et al: Clinical diagnosis of heterozygous dystrophin gene deletions by fluorescence in situ hybridization. Neuromusc Disord 1998; 8: 447-452.

12 Chamberlain JS, Gibbs RA, Ranier JE, Nguyen PN, Caskey CT: Deletion screening of the Duchenne muscular dystrophy locus via multiplex DNA amplification. Nucleic Acids Res 1998; 16: 11141-11155.
13 Beggs AH, Koenig M, Boyce FM, Kunkel LM: Detection of $98 \%$ of DMD/BMD gene deletions by polymerase chain reaction. Hum Genet 1990; 86: 45-48.

14 Feinberg AP, Vogelstein B: A technique for radiolabeling DNA restriction endonuclease fragments to high specific activity. Anal Biochem 1984; 132: 6-13.

15 Sambrook J, Fritsch EF, Maniatis T: Molecular Cloning: A Laboratory Manual, 2nd edn. Cold Spring Harbor Laboratory Press, 1989.

16 Ligon AH, Beaudet AL, Shaffer LG: Simultaneous, multilocus FISH analysis for detection of microdeletions in the diagnostic evaluation of developmental delay and mental retardation. Am J Hum Genet 1997; 61: 51-59.

17 Knight SJL, Horsley SW, Regan R et al: Development and clinical application of an innovative fluorescence in situ hybridization technique which detects submicroscopic rearrangements involving telomeres. Eur J Hum Genet 1997; 5: 1-8.

18 Pinkel $D$, Segraves $R$, Sudar $D$ et al: High resolution analysis of DNA copy number variation using comparative genomic hybridization to microarrays. Nat Genet 1998; 20: 207-211. 